Editorial

\title{
Clinic or home blood pressure: what to choose?
}

Volume 5 Issue 4 - 2016

\author{
Aurelio Leone ${ }^{1,2,3}$ \\ 'Fellow of the American Society of Hypertension (FASH), USA \\ ${ }^{2}$ Fellow of the Royal Society for Promotion of Health (FRSPH), \\ UK \\ ${ }^{3}$ Editor in Chief of the Journal of Cardiology \& Current \\ Research, USA
}

A correct approach to the treatment of hypertension closely depends on the blood pressure (BP) values to be reduced, ${ }^{1-3}$ because, commonly, lowering BP is the goal to be achieved, when possible, by changes in lifestyle, preventive measures and, of course, antihypertensive drugs. ${ }^{4,5}$ There is a need of accurate measurements of BP, which show to be a parameter to be correctly assessed in an attempt to choose those therapeutic and preventive approaches to fight hypertension. Thus, there is evidence that these measures widely vary among the different individuals and, therefore, should be related to $\mathrm{BP}$ values.

Significant changes in what was the normal BP values have been frequently observed in the last years, ${ }^{6-8}$ so that achieving the optimal BP target, delayed because of controversial opinions, shows nowadays promising results. From values of $140 / 85 \mathrm{mmHg}$ of a recent past, currently BP of $130 / 80 \mathrm{mmHg}$ or less $(120<80 \mathrm{mmHg})$ in case of other cardiovascular risk factors associated are the more common outcome.

Two methods for BP assessment are currently used to determine BP, respectively clinic and home BP measurements, because Ambulatory BP Monitoring, undoubtedly the most useful way to establish the true outcome of BP, ${ }^{9}$ cannot be carried out on large samples of populations for evident difficulties with its large-scale use.

Therefore, clinic or home BP determination to approach the true control of this parameter?

It is worth noting that a correct BP measurement continues to be one of the most important factor in clinical medicine since hypertension is a major risk factor for coronary heart disease, stroke, and renal failure, and affects approximately one-third of the world population. In addition, recently the condition of "prehypertension," that is, people with blood pressures at the high end of the normal range, carefully defined, makes the BP value assessment, particularly important in an attempt to control and/or avoid that a high value in the close future can be observed. ${ }^{10}$

Clinic BP measurement is primarily conducted in inpatients by the physicians or health staff in hospitals and/or medical offices. Once BP values are assessed, these parameters are commonly identified and considered to be the true BP of the individual. On the contrary, home BP measurement is personally conducted by the subject who checks his BP, which, therefore, depends on subject ability.

There is evidence that the two methods differ and, therefore, a comparison related to the characteristics of the used devices and individual ability to perform these techniques need. When a correspondence of measurements is stably observed, the BP values may be considered reliable and the hypertensive individuals can surely measure home BP for their convenience.

Current data on the subject permit very interesting conclusions. ${ }^{11}$

Firstly, it is worth noting that effective and safe devices precisely calibrated, which are able to compete with the classic Sphygmomanometers used for clinic BP assessment, are on sale. Secondly, evidence indicates that the large majority of hypertensive subjects is nowadays able to assess the home BP and, therefore, could be invited to apply successfully this method. However, according to a paper ${ }^{11}$ that underlined that neither clinic nor home measurements have sufficient sensitivity or specificity to be recommended as a single diagnostic test to define an individual as a hypertensive patient, there is evidence that efforts to diagnose carefully if elevated BP really exists should precede the question of what type of measurement, clinic or home BP, is more effective to be used.

My personal opinion is when BP is carefully measured and there are little differences in the values by a method rather than another one, a patient can be invited to use the most comfortable type of measurement, taking into account that a numerical correction factor may be always analyzed and introduced to compare a little gap existing. In addition, since the same individual checks his BP is obvious to keep in mind a single and always same correction may be currently applied.

The basic concept that should be carefully taken into account is a hypertensive patient makes the measurements of BP familiar with him rather than avoid to control frequently his BP with the risk of cardiovascular complications that a correct approach to the rules established for preventing the damages due to hypertension could reduce in the rate. In addition, some factors usually influence the results obtained by clinic BP because of subjects who do not bear any contact with medical structures. Therefore, once assessed the BP values, home measurements should be encouraged primarily for those people having significant differences between clinic and home BP depending on several conditions related to individual characteristics like masked hypertension, white coat hypertension and/or other commonly coming up, which can explain the observation of dissimilar values in the two methods. 


\section{Acknowledgments}

None.

\section{Conflicts of interest}

Author declares that there is no conflict of interest.

\section{References}

1. Leone A. The Control of Blood Pressure Worldwide: A Glimmer of Success. J Cardiol Curr Res. 2015;3(5):00117.

2. Leone A, Landini L, Leone A. Epidemiology and costs of hypertensionrelated disorders. Curr Pharm Des. 2011;17(28):2955-2972.

3. Booth J. A short history of blood pressure measurement. Proc R Soc Med. 1977;70(11):793-799.

4. Moser M, Roccella EJ. The treatment of hypertension: a remarkable success story. J Clin Hypertens. 2013;15(2):88-91.

5. James PA, Oparil S, Carter BL, et al. Evidence-bases guidelines for the management of high blood pressure in adults: Report from the Panel Members Appointed to the Eight Joint National Committee (JNC 8). JAMA. 2014;311(5):507-520.

6. Verdecchia P. Reference values for ambulatory blood pressure and selfmeasured blood pressure based on prospective outcome data. Blood Press Monit. 2001;6(6):323-327.
7. Barba G, Buck C, Bamman K, et al. Blood pressure reference values for European non-overweight school children: The IDEFICS study. Int $J$ Obes (Lond). 2014;38(Suppl 2):S48-S56.

8. Ogihara T, Nakao K, Fukui T, et al. The optimal target blood pressure for antihypertensive treatment in Japanese elderly patients with highrisk hypertension: a subanalysis of the Candesartan Antihypertensive Survival Evaluation in Japan (CASE-J) trial. Hypertens Res. 2008;31(8):1595-1601.

9. Leone A. Evidence of Impaired Night-Response of Blood Pressure in Hypertensive Smokers. An Update. J Cardiol Curr Res. 2015;4(2):00134.

10. Pickering TG, Hall JE, Appel LJ, et al. Recommendations for Blood Pressure Measurement in Humans and Experimental Animals. Part 1: Blood Pressure Measurement in Humans: A Statement for Professionals From the Subcommittee of Professional and Public Education of the American Heart Association Council on High Blood Pressure Research. Hypertension. 2005;45:142-161.

11. Hodgkinson J, Mant J, Martin U, et al. Relative effectiveness of clinic and home blood pressure monitoring compared with ambulatory blood pressure monitoring in diagnosis of hypertension: systematic review. BMJ. 2011;342:d3621. 